\title{
Band structure and many body effects in graphene
}

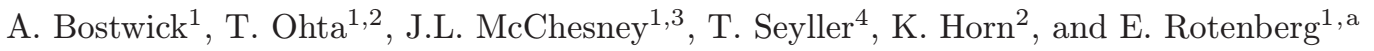 \\ 1 Advanced Light Source, E.O. Lawrence Berkeley National Laboratory, Berkeley CA 94720, USA \\ 2 Department of Molecular Physics, Fritz-Haber-Institut der Max-Planck-Gesellschaft, \\ Faradayweg 4-6, 14195 Berlin, Germany \\ 3 Department of Physics, Montana State University, PO Box 173840, Bozeman, MT 59717-3840, USA \\ ${ }^{4}$ Institut für Physik der Kondensierten Materie, Lehrstuhl für Technische Physik, \\ Universität Erlangen-Nürnberg, Erwin-Rommel-Straße 1, 91058 Erlangen, Germany
}

\begin{abstract}
We have determined the electronic bandstructure of clean and potassium-doped single layer graphene, and fitted the graphene $\pi$ bands to a one- and three-near-neighbor tight binding model. We characterized the quasiparticle dynamics using angle resolved photoemission spectroscopy. The dynamics reflect the interaction between holes and collective excitations, namely plasmons, phonons, and electron-hole pairs. Taking the topology of the bands around the Dirac energy for $n$-doped graphene into account, we compute the contribution to the scattering lifetimes due to electron-plasmon and electron phonon coupling.
\end{abstract}

\section{Introduction}

Graphene, a single layer of carbon atoms arranged in a honeycomb lattice, is the building block of $\mathrm{C}_{60}$, nanotubes, graphite, and other mesoscopic carbon systems. As such, graphene is a model system whose properties should be studied in order to understand exotic phenomena in all of carbon systems. It is also interesting in its own right since graphene has the potential to play an important role in future electronic technologies, and may display its own interesting fundamental two-dimensional (2D) phenomena reflected in transport measurements. The recent isolation of high-quality, few-layer graphene by exfoliation [1,2] and by epitaxial growth [3-8] has led to an explosion of theoretical and experimental studies of graphene.

The carrier dynamics can be explored directly using angle-resolved photoemission spectroscopy (ARPES), which determines the lifetime and mass renormalization of the carriers. In this paper, we present ARPES measurements of single layer graphene films grown on $\mathrm{SiC}$ substrates as a function of potassium doping. The $\pi$ bands are fitted to a tight-binding (TB) model, which simulates the bands very well except in the vicinity of the Dirac and Fermi levels $\left(E_{\mathrm{D}}\right.$ and $\left.E_{\mathrm{F}}\right)$, where the bands are renormalized by many-body interactions, namely electronplasmon, electron-electron, and electron-phonon interaction. These renormalizations turn out to be strongly affected by doping, which we determined by measuring the bands as a function of $n$-doping by potassium adsorption.

\section{Experimental}

Single layers of graphene were prepared by etching a $6 \mathrm{H}-\mathrm{SiC}(0001)$ substrate $(n$-type with a nitrogen concentration of $(1.5 \pm 0.5) \times 10^{18} \mathrm{~cm}^{-3}$ from SiCrystal AG) in hydrogen $(P=1 \mathrm{bar}$,

\footnotetext{
a e-mail: erotenberg@lbl.gov
} 
$T=1550^{\circ} \mathrm{C}, t=30 \mathrm{~min}$ ) followed by annealing at $1150^{\circ} \mathrm{C}$ for 4 minutes by direct current heating in pressure better than $1 \times 10^{-10}$ Torr. After preparation, the samples were transferred in vacuo to a liquid He-cooled cryostat, and the samples were cooled to around $20 \mathrm{~K}$ for ARPES measurements. The ARPES measurements were conducted at a pressure better than $2 \times 10^{-11}$ Torr.

The growth and ARPES measurements were conducted at the Electronic Structure Factory endstation at beamline 7.01 at the Advanced Light Source, which was equipped with a hemispherical electron energy analyzer (SES-R4000), using a photon energy $h \nu=94 \mathrm{eV}$ with an overall energy resolution of $\sim 25 \mathrm{meV}$. The samples were $n$-doped by adsorption of submonolayer amounts of potassium atoms at low temperature, which were deposited by commercial (SAES) getter sources. The angular emission patterns were converted to momentum space units by geometric transform. The absolute momentum scale in ARPES is difficult to determine exactly since it is sensitive to an inner potential parameter. In this paper, we have chosen this inner potential to scale the band structures to the distance $\Gamma K=1.703 \AA^{-1}$ or equivalently, a C-C bond length of $1.42 \AA$ or lattice constant $a=2.46 \AA$.

\section{Band structure and tight binding models}

Figure 1(a) shows the $\pi$ band structure of as-prepared graphene taken along principle directions of the graphene Brioullin zone (BZ). The measurements are extracted from a larger data set which samples momenta $\mathbf{k}$ throughout the 2D BZ. Our purpose in this section is to develop a tight-binding fit to the bandstructure, including the slowly-varying effects of many-body interactions on the overall bandwidth, but ignoring those many-body interactions which affect the detailed shape of the bands near $E_{\mathrm{F}}$. We first consider a simple single near neighbor $(\mathrm{NN})$ tight binding (TB) model as presented by Saito [9] and generalized to third-NN by Reich [10]. The first-NN bandstructure can be written in closed form as

$$
E(\mathbf{k})=\frac{\epsilon_{2 p} \pm \gamma_{0} w(\mathbf{k})}{1 \pm s_{0} w(\mathbf{k})}
$$

where

$$
w(\mathbf{k})=\sqrt{1+4 \cos \left(\sqrt{3} a k_{y} / 2\right) \cos \left(a k_{x} / 2\right)+4 \cos ^{2}\left(a k_{x} / 2\right)} .
$$

Here, $\gamma_{0}$ and $s_{0}$ are the NN hopping and overlap integrals, respectively, and $\epsilon_{2 p}$ is chosen to adjust the Fermi level $E_{\mathrm{F}}$ to the experimental value relative to $E_{\mathrm{D}}$. Previously, we neglected $s_{0}$ when comparing the model equation (1) to the data [8]. Here we show a systematic fit of the bands along the principle directions (figure 1(a)) including the overlap term, with the fitting parameters summarized in Table 1. Since for clean graphene, the states above the Dirac energy $E_{\mathrm{D}}$ are mostly unoccupied, the fit is only taken over the bands below $E_{\mathrm{D}}$. While the first-NN TB fit models the overall bandwith reasonably well, the detailed curvature of the bands is not well modelled, nor are the energies of the bottom of the $\pi$ band or of the saddle point at the M point of the BZ. This is because additional nearest neighbor hopping and overlap must be considered to get a more accurate fit.

Reich et al. evaluated the band structure from first principles, and constructed a third-NN TB model as a fit to the first principles calculation [10]. Their model is shown for comparison to the data in figure 1(a), which has been aligned to the bottom of the $\pi$ bands at $\Gamma$. This model underestimates the observed bandwidth (below $E_{\mathrm{D}}$ ) by around $11 \%$. A similar mismatch has also been seen for graphite, which was attributed to self-energy effects due to electron-electron interaction [11]. In order to present a more useful TB fit, we encapsulate this band widening, and other deviations of the bands from Reich's model, with an empirical fit of the bands to the graphene measurements (figure 1(a)). This fit is now seen to be quite satisfactory throughout a wide range of energies, and captures not only the bandwidth but also does a good job to match the detailed shape of the bands. This good agreement comes at the expense of having a rather larger hopping parameter $\left(\gamma_{0}=-5.13\right)$ than generally expected. 


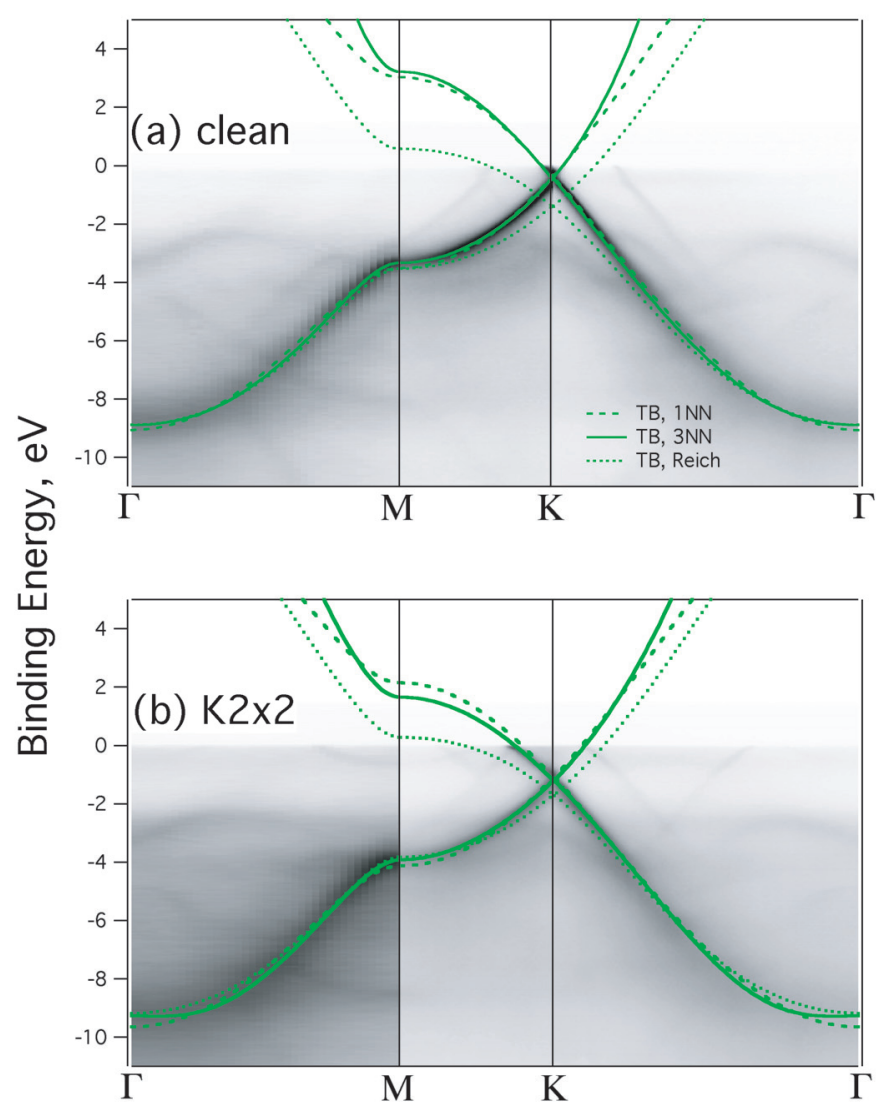

Fig. 1. Experimental and tight binding band structures for pure and $K-(2 \times 2)$ graphene. The bands for the principle directions of the graphene Brioullin zone (see inset, figure 2) are shown for (a) clean graphene and (b) $K-(2 \times 2)$ graphene as greyscale images (darker $=$ more intense). The best-fit tight binding bands are shown for one and three-NN models (dashed and solid, respectively). The third-NN model by Reich is shown for reference (dotted lines).

One must be careful to consider the doping level of the graphene, since we observed the TB parameters to be doping-dependent. To see the effect of doping, we deposited potassium atoms at $T=100^{\circ} \mathrm{K}$ until a sharp $2 \times 2$ reconstruction was observed. This reconstruction corresponds in stoichiometry and (we believe) arrangement to a single bilayer of the graphite intercalation compound $\mathrm{KC}_{8}$.

The data and comparisons to Reich's third-NN fit and our first- and third-NN fits, are shown in figure 1(b). The most prominent differences compared to clean graphene are (1) that the slope (or band velocity) near $E_{\mathrm{D}}$ has been reduced in the doped film, and now agrees reasonably well with that by Reich, and (2) the shape of the bands is somewhat different. Especially notable is the flattening of the bottom of the $\pi$ bands around $\Gamma$. This flattening cannot be accounted for except by higher terms, and hence is missing in the first-NN fit. Since this flattening is missed by the Reich model, it must be due to some self-energy corrections, which would be interesting to explore further but which are beyond the scope of this paper.

One should note that similar to the clean graphene case, the third-NN fit in figure 1(b) was carried out only for data below $E_{\mathrm{D}}$. Despite the large number of terms, the data above $E_{\mathrm{D}}$ seem to be well-accounted for, apart from small deviations to be discussed below. This suggests that we might be able to trust the extrapolated bands above $E_{\mathrm{F}}$ as well. In that case, we can see that in the doped $\mathrm{K}$ case, we can observe a relative narrowing of the unoccupied bands around the $\mathrm{M}$ saddle point. This trend is continued for even higher doping than is presented here [12]. 
Table 1. Tight binding parameters for one- and three-NN fits of the data in figure 1, also shown are the fits to theory by Reich [10].

\begin{tabular}{ccccccc}
\hline Sample & $\gamma_{0}[\mathrm{eV}]$ & $s_{0}$ & $\gamma_{1}[\mathrm{eV}]$ & $s_{1}$ & $\gamma_{2}[\mathrm{eV}]$ & \multicolumn{1}{c}{$s_{2}$} \\
\hline clean, 1-NN & -3.28 & 0.0425 & & & & \\
clean, 3-NN & -5.13 & -0.148 & 1.70 & -0.0948 & -0.418 & 0.0743 \\
$K-(2 \times 2), 1-N N$ & -3.17 & 0.033 & & & & \\
$K-(2 \times 2), 3-N N$ & -3.64 & -0.238 & 1.33 & -0.0537 & -0.344 & 0.0626 \\
Reich, 3-NN & -2.97 & 0.073 & -0.073 & 0.018 & -0.33 & 0.026 \\
\hline
\end{tabular}

\section{Many body interactions at low energy scales}

With the discovery of superconductivity in carbon nanotubes (CNTs) [13,14], alkali-metaldoped $\mathrm{C}_{60}$ crystals [15], and graphite intercalation compounds [16-18] (GICs) with relatively high transition temperatures, there is a strong interest in the influence of many-body interactions on the electron dynamics of graphite and related materials. Graphene and graphite are also interesting because their carriers can be described as relativistic Fermions through a formal equivalence of the wave equation with the relativistic Dirac equation $[1,2,19]$. Departure of the electron dynamics from Fermi liquid behavior in graphite has also been attributed to the special shape of the graphene bandstructure [20,21]. It is interesting to ask whether any such departure is related to the massless character of the Dirac fermions.

With the ARPES technique, we can derive not only the bandstructure as discussed in section 3, but also the quasiparticle dynamics. We found that the bands are strongly renormalized near $E_{\mathrm{D}}$ and $E_{\mathrm{F}}$ by electron-electron, electron-phonon, and electron-plasmon coupling effects. The latter coupling is particularly interesting because it distorts the bands near $E_{\mathrm{D}}$. Figure $2(\mathrm{a})$ shows a magnified view of the bands measured along a line (the vertical double arrow in the inset) through the $\mathrm{K}$ point. The predicted (bare) bands are nearly perfectly linear and mirrorsymmetric with respect to the $\mathrm{K}$ point according to equation (1). The actual bands deviate from this prediction in two significant ways: first, at a binding energy $\sim 200 \mathrm{meV}$ below $E_{\mathrm{F}}$, we observe a sharpening of the bands accompanied by a slight kink in the bands' dispersions. We attribute this to renormalization of the electron bands near $E_{\mathrm{F}}$ by coupling to phonons, as discussed later.

Second, linear extrapolations of the lower bands (dashed lines in figure 2(a)) do not pass through the upper bands, demonstrating that the bands do not pass smoothly through $E_{\mathrm{D}}$ as equation (1) predicts. This is observed more easily for data acquired along the orthogonal direction through the $\mathrm{K}$ point (figure 2(e)), where a favorable matrix element effect [22] suppresses one of the two bands. We observe an additional kink near $E_{\mathrm{D}}$, which we propose is caused by many-body interactions.

The deviations from the bare band are sensitive to doping, which we varied by adsorbtion of potassium atoms. The evolution of the band structure upon doping is followed in figure 2(b)(d) and along the orthogonal direction in figure 2(f)-(h). Similar to graphite, doping graphene by $\mathrm{K}$ deposition shifts the bands more or less rigidly to higher binding energy [23]. While the energy of the kink at $200 \mathrm{meV}$ does not change, the second kink strengthens and follows $E_{\mathrm{D}}$ with doping, demonstrating that it is associated with electrons with energy near $E_{\mathrm{D}}$. The effect of this kink on the bandstructure is significant: at high doping, a curve fit of the band positions (figure $2(\mathrm{~d})$ ) shows that $E_{\mathrm{D}}$ has been shifted towards $E_{\mathrm{F}}$ by $130 \mathrm{meV}$ from the single-particle prediction.

In the quasiparticle scheme, ARPES measures the spectral function, expressed in terms of the complex self energy $\Sigma(\mathbf{k}, \omega)$, as $[24,25]$

$$
A(\mathbf{k}, \omega)=\frac{|\operatorname{Im} \Sigma(\mathbf{k}, \omega)|}{\left(\omega-\omega_{\mathrm{b}}(\mathbf{k})-\operatorname{Re} \Sigma(\mathbf{k}, \omega)\right)^{2}+\operatorname{Im} \Sigma(\mathbf{k}, \omega)^{2}}
$$

where $\omega$ is the quasiparticle energy, and $\omega_{\mathrm{b}}(\mathbf{k})$ is the unrenormalized, or "bare" band dispersion in the absence of many-body effects. The form of equation (3) is that of a Lorentzian whose 

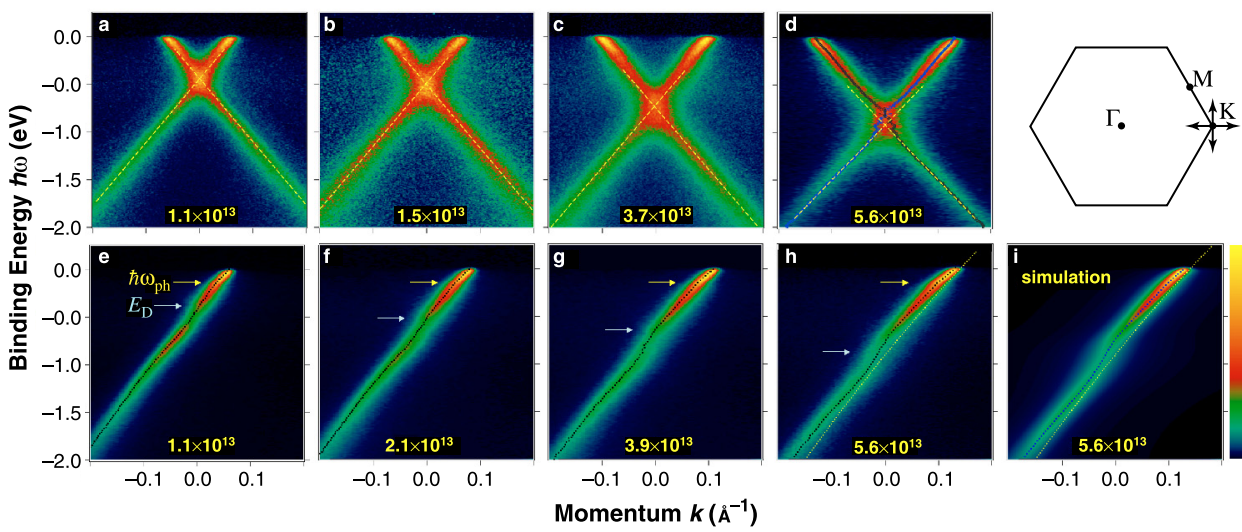

$\begin{array}{lll}-0.1 & 0.0 & 0.1\end{array}$

Momentum $k\left(\AA^{-1}\right)$

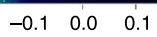

$\begin{array}{lll}-0.1 & 0.0 & 0.1\end{array}$

Fig. 2. The bandstructure of graphene near the Fermi Level. (a)-(d) Experimental energy bands along the vertical double-arrow in the inset as a function of doping. The dashed lines are an extrapolation of the lower bands (below the Dirac crossing energy $E_{\mathrm{D}}$ ). The electron density (per $\mathrm{cm}^{2}$ ) is indicated on each panel. (e)-(h) Bandmaps for similar dopings acquired along the horizontal double arrow in the inset. The non-linear, or "kinked" dispersion of the bands is evident from the fitted band position (dotted lines) (i) The simulated spectral function, calculated using only the bare band (yellow dotted line) and $\operatorname{Im} \Sigma$ derived from the data in panel h. From [8].

position (relative to $\omega_{\mathrm{b}}$ ) and width are given by the real and imaginary parts of the self-energy function $\Sigma(\mathbf{k}, \omega)$. This function plays the same role for the quasiparticles as the complex index of refraction plays for photons in an interacting medium. Thus, it encodes both the scattering rate and the renormalization of the band dispersion in its imaginary and real parts, respectively.

In the k-independent approximation $[25,26](\Sigma(\mathbf{k}, \omega) \approx \Sigma(\omega)), \operatorname{Im} \Sigma(\omega)$ is proportional to the Lorentzian linewidth of the so-called momentum distribution curve (MDC) $A(\mathbf{k}, \omega)$, which is a horizontal cut of the spectral function taken at constant $\omega$. Like the complex index of refraction, $\operatorname{Re} \Sigma(\omega)$ is readily computed from $\operatorname{Im} \Sigma(\omega)$ through a Hilbert transform (to satisfy causality), and the full spectral function $A(\mathbf{k}, \omega)$ can be reconstructed using the computed $\operatorname{Re} \Sigma(\omega)$ and compared to experiment. Such a reconstruction for one doping is shown in figure 2(i); it is in excellent agreement with the data (figure $2(\mathrm{~h})$ ) from which $\operatorname{Im} \Sigma$ was obtained. This shows that the kinks in the bands originate not from details of the single-particle bandstructure, but rather from many-body interactions, providing strong support for the quasiparticle picture in graphene.

The observed kink structure is therefore derived from a complicated $\omega$-dependence of the observed scattering rate (proportional to the MDC linewidths shown in figure 3) as a function of doping. To model this behavior, we consider three processes: decay of the carriers by phonon emission, by electron-hole $(e-h)$ pair generation, and by emission of collective charge excitations (plasmons) via electron-plasmon $(e-p l)$ coupling. (Impurity scattering, a fourth contributing process, is a relatively small contribution which can be neglected.) By summing up all the momentum- and energy-conserving decay events as a function of hole energy $\omega$, we can show that the three principle decay processes contribute differently to the lifetime in regions I-IV as identified in figure 3 .

Such a calculation for the total scattering rate, together with the individual contributions from $e-p h, e-h$ and $e-p l$ processes, is shown in figure 3. This calculation is for a sample with $n=5.6 \times 10^{13} \mathrm{~cm}^{-2}$ and compares favorably to the experimental MDC width for that doping; similar agreement can be obtained for the other dopings as well. The predicted dip at $E_{\mathrm{D}}$ is an artefact of the simplicity of our model, which does not consider interactions between the plasmons and the Fermi liquid excitations.

Now we discuss the different decay processes in turn. We attribute the kink near $E_{\mathrm{F}}$ to electron-phonon $(e-p h)$ coupling as described previously for metals [27-29], graphite [30,31] and (possibly) high- $T_{c}$ superconductors $[32,33]$. E-ph coupling is expected at this energy scale considering the known phonon spectrum [34]. In this process, photoholes decay by phonon 


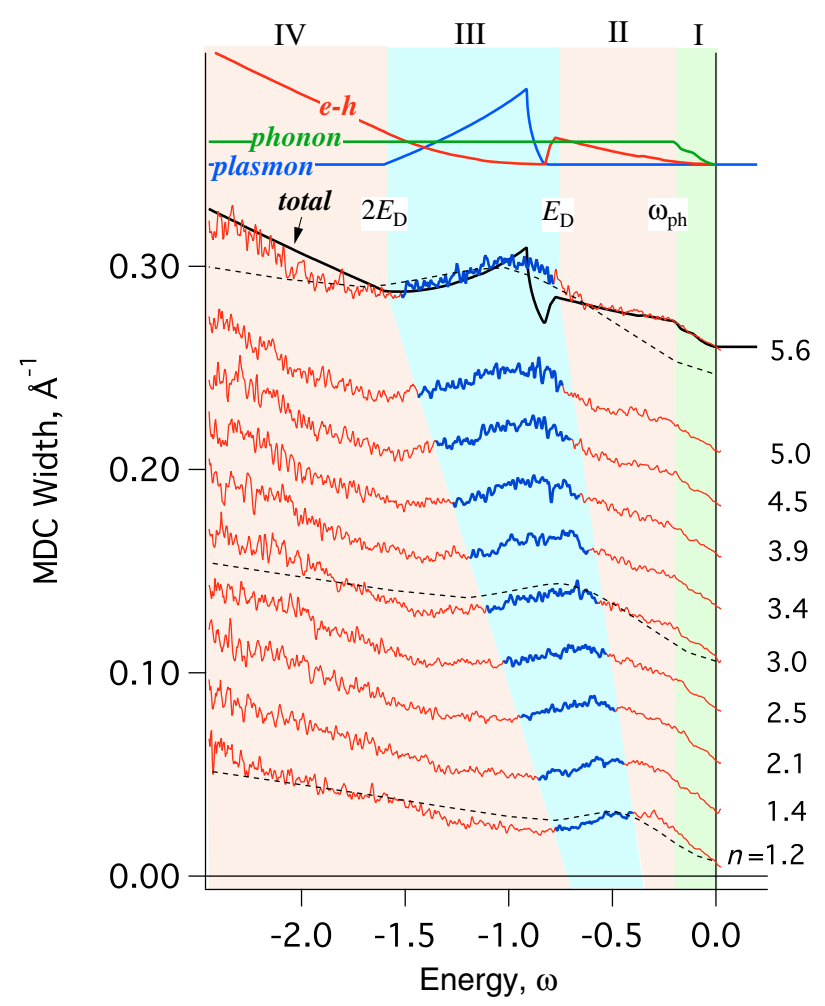

Fig. 3. MDC Widths of Carriers in Graphene. Measured spectral linewidth (assumed proportional to scattering rate and $\operatorname{Im} \Sigma$ ) for graphene, derived by performing a line shape analysis of MDCs for each binding energy as a function of doping $n\left(10^{13} \mathrm{~cm}^{-2}\right)$. Each trace is shifted upward by $0.025 \AA^{-1}$. The simulated total scattering contribution (black line) and the partial contributions due to decay into phonons (green), $e-h$ pairs (red) and plasmons (blue) are calculated for the highest doped sample. These interactions contribute differently in regions I-IV defined as follows: (I) the phonon energy scale $\omega_{\mathrm{ph}}<\omega<0$, (II) the Dirac energy scale $\omega_{\mathrm{D}}<\omega<0$, (III) $2 \omega_{\mathrm{D}}<\omega<\omega_{\mathrm{D}}$, (IV) $\omega<2 \omega_{\mathrm{D}}$. The recently calculated MDC widths by Hwang et al. for three representative dopings are shown for comparison ([38], dashed lines). Adapted from [8].

emission (see figure 4(a)). Using the graphite phonon density of states [35], we calculated the $e-p h$ contribution to $\operatorname{Im} \Sigma$ (figure 3, green curve) with the standard formalism [36] and find an $e$-ph coupling constant $\lambda \approx 0.3$. Although this is a factor of 5 larger than predicted [37] for $n=5.6 \times 10^{13} \mathrm{~cm}^{-2}$, comparison with the experimental data shows that this provides an accurate description of $\operatorname{Im} \Sigma$ in region I. The observed increase of the kink's strength with $n$ (see figure $2(\mathrm{e})-(\mathrm{h})$ ) is expected from the increase in the size of the Fermi surface, although the $200 \mathrm{meV}$ energy scale remains constant because the K atoms should not significantly alter the phonon spectrum of graphene.

Consider now the decay of the photohole by excitation of an electron from below to above $E_{\mathrm{F}}$ thereby creating an $e-h$ pair. In Landau's Fermi liquid (FL) model, the decay rate increases as $\sim \omega^{2}$ away from $\omega=0$, reflecting the growing number of possible excitations that satisfy momentum and energy conservation. However, the linear dispersion of the graphene bands and the presence of the Dirac crossing drastically modify this picture. A hole just above $E_{\mathrm{D}}$ can easily decay through many possible $e-h$ creation events, for example as in figure 4 (b), and we find a similar $\omega^{\alpha}(\alpha \sim 1.5)$ dependence of $\operatorname{Im} \Sigma$ in regions I-II as in FL theory. But a hole decay originating at $\omega$ just below $E_{\mathrm{D}}$ has few final states with sufficient momentum transfer to excite an $e-h$ pair (figure $4(\mathrm{c})$ ). This causes a sharp reduction in the scattering rate in region III. Only for energies in region IV does $e-h$ pair generation become favorable (e.g. figure 4(d)). 

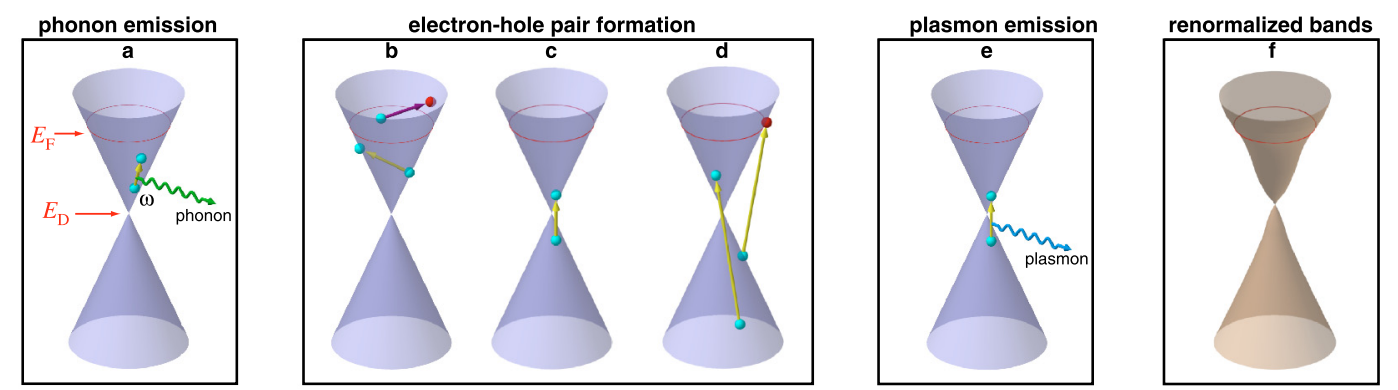

Fig. 4. Decay and Scattering Processes in Graphene. (a) The energy/momentum diagrams for decay processes scattering of a photohole, initially created at energy $\omega$, decaying by a emission of a phonon. (b)-(d) Spontaneous generation of an electron-hole pair near the Fermi level $E_{\mathrm{F}}$ for photohole energy satisfying (b) $\left(\omega>\omega_{\mathrm{D}}\right)$, (c) $\left(2 \omega_{\mathrm{D}}<\omega<\omega_{\mathrm{D}}\right)$, which can generate no possible $e$ - $h$ pair as drawn, and (d) $\left(\omega<2 \omega_{\mathrm{D}}\right)$. (e) emission of a plasmon $\left(2 \omega_{\mathrm{D}}<\omega<\omega_{\mathrm{D}}\right)$. (f) The net effect of these processes is to distort the bare bands to the renormalized bands (shown in tan). From [8].

The $e-h$ and $e-p h$ processes can explain the observed MDC widths in regions I, II, and IV. In region III, however, decay by $e-h$ pair creation is practically not allowed yet the observed scattering rate has a peak rather than a dip (highlighted in blue in figure 3). This peak may be explained by decay through plasmon emission. In graphene, the charge carriers near the $\mathrm{K}$ point have zero effective mass and travel like photons at constant speed $\mathrm{c}^{*}$, but unlike photons, they have charge and are therefore subject to collective oscillations such as plasmons. Although a full treatment of the $e-p l$ interaction is difficult near the Dirac point, a simple model suffices to explain how $e-p l$ coupling can enhance the scattering rate below $E_{\mathrm{D}}$.

Ordinary two-dimensional plasmons have a dispersion relationship in the long-wavelength limit $[39]$

$$
\omega_{\mathrm{pl}}(q)=\sqrt{4 \pi n e^{2} q / m(1+\epsilon)}
$$

where $q$ is the plasmon momentum, $m$ is the carrier mass, and $\epsilon \sim 10$ is the dielectric constant. Although plasmons in principle exist in the domain $0<q<\infty$, in practice they propagate freely up to a critical momentum $q<q_{\mathrm{c}}$ due to Landau damping (plasmon decay into electron-hole pairs) [40].

For graphene, the rest mass $m$ is zero near $E_{\mathrm{D}}$ but the relativistic mass $m_{\mathrm{r}}=E / c^{* 2}$ is on the order [1] of $0.1 \mathrm{~m}_{\mathrm{e}}$ and can be used to set the plasmon energy scale $\omega_{\mathrm{pl}}$. Since the plasmon spectrum $\omega_{\mathrm{pl}}(q)$ rises steeply over a short, finite range of $q$, decay of the photohole into plasmons becomes kinematically possible only for hole decays originating just below $E_{\mathrm{D}}$ (figure $4(\mathrm{e})$ ).

The simple plasmon spectrum equation (4) is illustrated in figure 5, calculated using the experimentally determined carrier masses [1] for a range of dielectric constants (3), (6) and (10). Also shown is the recent calculation of the plasmon spectrum [38]. As expected, when the plasmon energy-momentum is outside the range of $e-h$ excitations (shaded region) the simple model equation (4) is a very good approximation to the computed plasmon spectrum in the relevant excitation region at small $q$. (Similar plasmon dispersions have also been presented at lower dopings $[41,42]$ and for a graphene bilayer [43].)

Given the plasmon dispersion relation we can easily sum up the possible plasmon decays as a function of $\omega$ (figure 3, blue curve), which is proportional to the scattering rate. We find a peak located just below $E_{\mathrm{D}}$, whose width and intensity scales with $E_{\mathrm{D}}$. A peak following these trends is clearly observed in the experimental data (highlighted in blue in figure 3). A more detailed calculation by Hwang et al. has also confirmed the existence of this $e$ - $p l$ scattering peak [38]. Their calculation of the $e-p l$ and $e$-e contributions to the scattering rate is shown for three dopings as the dashed lines in figure 3.

Previously, $e-p l$ coupling was shown to affect the unoccupied bands of a $3 \mathrm{D}$ metal at the large plasmon energy scale $(\sim 20 \mathrm{eV})[44]$. But $e$ - $p l$ coupling at small energy scales is normally forbidden for 2- and 3D electron gases (except for the special case of layered electron gases) [20] so this is a unique instance where $e-p l$ coupling is kinematically allowed for a pure $2 \mathrm{D}$ system. 


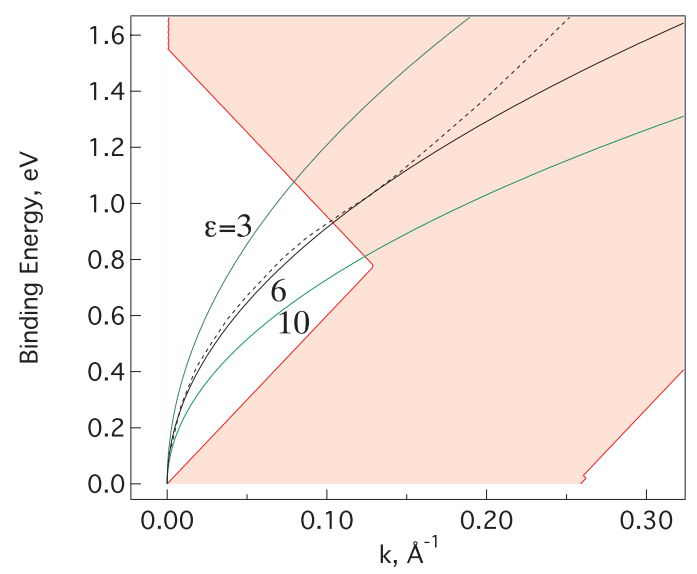

Fig. 5. Energy diagram of the electronic excitations in graphene. The shaded red region shows the possible $e-h$ pair excitations for graphene, computed for a conical band structure with $n=5.6 \times$ $10^{13} \mathrm{~cm}^{-2}$. The lines show the plasmon dispersion calculated for dielectric constants 3,6 , and 10 using equation (4) together with the relativisitic mass taken from transport measurements $[1,2]$. The calculated plasmon dispersion of Hwang et al. ([38], dashed line) is shown for comparison. Adapted from $[8]$.

It is also remarkable because of the relativistic nature of the carriers and the strong role it plays in shifting the Dirac energy.

It is worth emphasizing that the model for the scattering rate has only four adjustable scaling factors: the $e-p h$ coupling constant $\lambda$, the absolute probabilities for $e-h$ pair creation and plasmon emission, and the screening constant $\epsilon$ which scales the Coulomb interaction. The other inputs are the experimentally determined band dispersion, the graphite phonon density of states, and the relativistic mass $m_{\mathrm{r}}$ which is taken from the literature [1].

These results show that the special condition of massless Dirac Fermions found in graphene does not preclude the validity of the quasiparticle picture - in fact the quasiparticle picture is valid over a spectacularly wide energy range - but it does induce novel $e-h$ and $e-p l$ decay processes. These result in strong modifications of the band dispersion, as schematically illustrated in figure 4(f). This distortion occurs not only near the Fermi level as in conventional metals, but also centered around the Dirac crossing energy $E_{\mathrm{D}}$. The effects we describe are not unique to high doping levels, but extrapolate all the way down to zero doping. Near this regime (already approached for the lowest dopings in figure 3(b)), the energy scales for $e-h, e-p l$ and $e$ - $p h$ decay processes overlap, and a unified treatment of all these interactions is necessary to reproduce the many-body effects. These conclusions apply as well to graphite, nanotubes, and other carbon materials with similar electronic structure.

\section{References}

1. K.S. Novoselov, E. McCann, S.V. Morosov, V.I. Fal'ko, M.I. Katsnelson, U. Zeitler, D. Jiang, F. Schedin, A.K. Geim, Nature 438, 192 (2005)

2. Y. Zhang, Y.W. Tan, H.L. Stormer, P. Kim, Nature 438, 201 (2005)

3. I. Forbeaux, J.M. Themlin, J.M. Debever, Phys. Rev. B 58, 16396 (1998)

4. C. Berger, Z. Song, T. Li, X. Li, A. Ogbazghi, R. Feng, Z. Dai, A.N. Marchenkov, E.H. Conrad, P.N. First et al., J. Phys. Chem. B 108, 19912 (2004)

5. C. Berger, Z. Song, X. Li, X. Wu, N. Brown, C. Naud, D. Mayou, T. Li, J. Hass, A. Marchenkov et al., Science 312, 1191 (2006)

6. E. Rollings, G.H. Gweon, S.Y. Zhou, B.S. Mun, J.L. McChesney, B.S. Hussain, A.V. Fedorov, P.N. First, W.A. de Heer, A. Lanzara, J. Phys. Chem. Solids 67, 2172 (2006)

7. T. Seyller, K.V. Emtsev, F. Speck, K.Y. Gao, L. Ley, Appl. Phys. Lett. 88, 242103 (2006)

8. A. Bostwick, T. Ohta, T. Seyller, K. Horn, E. Rotenberg, Nat. Phys. 3, 36 (2007) 
9. R. Saito, G. Dresselhaus, M.S. Dresselhaus, Physical Properties of Carbon Nanotubes (Imperial College Press, 1998)

10. S. Reich, J. Maultzsch, C. Thomsen, P. Ordejón, Phys. Rev. B 66, 035412 (2002)

11. C. Heske, R. Treusch, F.J. Himpsel, S. Kakar, L.J. Terminello, H.J. Weyer, E.L. Shirley, Phys. Rev. B 59, 4680 (1999)

12. J.L. McChesney, T. Ohta, B. Bostwick, T. Seyller, K. Horn, E. Rotenberg (2007) (submitted)

13. Z. Tang, L.Y. Zhang, N. Wang, X. Zhang, G.H. Wen, G.D. Li, J.N. Wang, C.T. Chan, P. Sheng, Science 292, 2462 (2001)

14. M. Kociak, A.Y. Kasumov, S. Guron, B. Reulet, I.I. Khodos, Y.B. Gorbatov, V.T. Volkov, L. Vaccarini, H. Bouchiat, Phys. Rev. Lett. 86, 2416 (2001)

15. A.F. Hebard, M.J. Rosseinsky, R.C. Haddon, D.W. Murphy, S.H. Glarum, T.T.M. Palstra, A.P. Ramirez, A.R. Kortan, Nature 350, 600 (1991)

16. N.B. Hannay, T.H. Geballe, B.T. Matthias, K. Andres, P. Schmidt, D. MacNair, Phys. Rev. Lett. 14, $225(1965)$

17. T.E. Weller, M. Ellerby, S.S. Saxena, R.P. Smith, N.T. Skipper, Nat. Phys. 1, 39 (2005)

18. V.J. Emery, S.A. Kivelson, H.Q. Lin, Phys. Rev. Lett. 64, 475 (1990)

19. D.P. DiVincenzo, E.J. Mele, Phys. Rev. B 29, 1685 (1984)

20. S.M. Xu, J. Cao, C.C. Miller, D.A. Mantell, R.J.D. Miller, Y. Gao, Phys. Rev. Lett. 76, 483 (1996)

21. G. Moos, C. Gahl, F. Fasel, M. Wolf, T. Hertel, Phys. Rev. Lett. 87, 267402 (2001)

22. E. Shirley, L. Terminello, A. Santoni, F.J. Himpsel, Phys. Rev. B 51, 13614 (1995)

23. P. Bennich, C. Puglia, P.A. Brühwiler, A. Nilsson, A.J. Maxwell, A. Sandell, N. Martensson, P. Rudolf, Phys. Rev. B 59, 8292 (1999)

24. A. Damascelli, Z. Hussain, Z.X. Shen, Rev. Mod. Phys. 75, 473 (2003)

25. A. Kaminski, H.M. Fretwell, New J. Phys. 7, 1367 (2005)

26. A.A. Kordyuk, S.V. Borisenko, A. Koitzsch, J. Fink, M. Knupfer, H. Berger, Phys. Rev. B 71, $214513(2005)$

27. T. Valla, A. Fedorov, P. Johnson, S. Hulbert, Phys. Rev. Lett. 83, 2085 (1999)

28. M. Hengsberger, D. Purdie, P. Segovia, M. Garnier, Y. Baer, Phys. Rev. Lett. 83, 592 (1999)

29. E. Rotenberg, J. Schaefer, S. Kevan, Phys. Rev. Lett. 84, 2925 (2000)

30. S.Y. Zhou, G.H. Gweon, A. Lanzara, Ann. Phys. 321, 1730 (2006)

31. K. Sugawara, T. Sato, S. Souma, T. Takahashi, H. Suematsu, Phys. Rev. Lett. 98, 036801 (2007)

32. T. Valla, A.B. Federov, P. Johnson, B.O. Wells, S. Hulbert, Q. Li, G.U. Gu, N. Koshizuka, Science 285, 2110 (1999)

33. A.A. Kordyuk, S.V. Borisenko, A. Koitzsch, J. Fink, M. Knupfer, H. Berger, Phys. Rev. B 71, $214513(2005)$

34. R. Al-Jishi, G. Dresselhaus, Phys. Rev. B 26, 4514 (1982)

35. L. Vitali, M.A. Schneider, K. Kern, L. Wirtz, A. Rubio, Phys. Rev. B 69, 121414 (2004)

36. G. Grimvall, The Electron Phonon Interaction in Metals (North Holland Publishing Company, 1981)

37. M. Calandra, F. Mauri, Phys. Rev. Lett. 95, 237002 (2005)

38. E.H. Hwang, B. Yu-Kuang Hu, S.D. Sarma, cond-mat/0612345 (2006)

39. F. Stern, Phys. Rev. Lett. 18, 546 (1967)

40. K.L. Kliewer, H. Raether, Phys. Rev. Lett. 30, 971 (1973)

41. O. Vafek, Phys. Rev. Lett. 97, 266406 (2006)

42. X.F. Wang, T. Chakraborty, Phys. Rev. B 75, 033408 (2007)

43. X.F. Wang, T. Chakraborty, Phys. Rev. B 75, 041404 (2007)

44. M. Hengsberger, D. Purdie, P. Segovia, M. Garnier, Y. Baer, Phys. Rev. Lett. 83, 592 (1999) 